\title{
Theaflavins decrease skeletal muscle wasting in disuse atrophy induced by hindlimb suspension in mice
}

\author{
Kenta Suzuki, ${ }^{1}$ Nayuta Hirashima, ${ }^{1}$ Yasuyuki Fujii,, ${ }^{1}$ Taiki Fushimi, ${ }^{1}$ Ayaka Yamamoto,, ${ }^{2}$ Tomoya Ueno, ${ }^{2}$ \\ Ryota Akagi, ${ }^{1}$ and Naomi Osakabe ${ }^{1, *}$ \\ 'Department of Bio-science and Engineering, Shibaura Institute of Technology, 307 Fukasaku, Munumaku, Saitama 337-8570, Japan \\ ${ }^{2}$ Division of Research and Development, Yaizu Suisankagaku Ind. Co. Ltd., 5-8-13 Kogawashinmachi, Yaizu, Shizuoka 425-8570, Japan
}

\begin{abstract}
We previously found that a single dose of theaflavins induced skeletal muscle metabolic changes. In this study, we examined the effect of theaflavins on disuse muscle atrophy model mice by hindlimb suspension. Mice were assigned to 4 groups; groundvehicle, ground-theaflavins, suspension-vehicle, and suspensiontheaflavins, dosed with theaflavins $(250 \mathrm{mg} / \mathrm{kg} /$ day $)$ for 2 weeks. The peak of myotube size of cross sectional area was significantly moved to the smaller side in the suspension-vehicle group compared with the ground-vehicle group, and these shifts were significantly reduced by the treatment with theaflavins in both soleus and extensor digitorum longus. The level of phosphorylated eukaryotic translation initiation factor 4E-binding protein (4EBP)-1, located downstream of the Akt/mTOR pathway, was significantly different between suspension-vehicle and suspension-theaflavins in soleus. The ratio of forkhead box $\mathrm{O}$ (FoxO) $3 a$ to phosphorylated FoxO3a significantly increased in soleus or tended to rise in extensor digitorum longus of suspension-vehicle group compared with ground-vehicle. In contrast, these changes were not observed in suspension-theaflavins group. These results suggested that theaflavins inhibited the progress of disuse muscle atrophy through modulation of protein metabolism.
\end{abstract}

Key Words: theaflavins, skeletal muscle atrophy, hindlimb suspension, FoxO3a

A meliorating skeletal muscle wasting is of high clinical importance in order to improve quality of life. ${ }^{(1)}$ Skeletal muscle disuse including sedentary lifestyle or bed rest can lead to muscle atrophy. ${ }^{(2)}$ Skeletal muscle is a dynamic, plastic tissue whose mass is regulated by the balance between the rate of muscle protein synthesis and breakdown. ${ }^{(3)}$

The prevention of disuse muscle atrophy requires a mechanistic understanding of the cellular signaling pathways that regulate both protein synthesis and degradation. ${ }^{(4)}$ Among the number of disuse models, hindlimb suspension leads to significant skeletal muscle atrophy. This model provides information on the morphological and molecular changes responsible for the mechanisms of disuseinduced muscle loss. ${ }^{(5,6)}$

Theaflavins are a type of polyphenols that are present in high concentrations in black tea. Four major theaflavins, theaflavin, theaflavin-3-O-gallate, theaflavin 3'-O-gallate, and theaflavin3,3 '-di-O-gallate, are formed from co-oxidation of selected pairs of green tea catechins during fermentation. ${ }^{(7,8)}$ During the processing of black tea, fresh leaves are crushed and allowed to undergo oxidation by polyphenol oxidase, resulting in the formation of polyphenolic dimers known as theaflavins. ${ }^{(8)}$

In previous meta-analyses, it was shown that consumption of black tea results in significant primary prevention of cardiovascular diseases by decreasing plasma LDL cholesterol levels and blood pressure..$^{(9,10)}$ We previously reported that repeated treatment with theaflavins showed a significant reduction of blood pressure in rodents. ${ }^{(11)}$ In addition, a single dose of theaflavins enhanced energy expenditure with an increase in proliferator-activated receptor gamma coactivator-1 $\alpha$ (PGC-1 $\alpha)$ transcription resulting in acceleration of AMP-activated protein kinase (AMPK) phosphorylation in mouse skeletal muscle. ${ }^{(12)} \mathrm{We}$ also reported previously that 10 weeks' supplementation of a theaflavins-rich capsule improved body composition, including a reduced ratio of body and subcutaneous fat and increased skeletal muscle percentage in healthy subjects. ${ }^{(13)}$ These results suggested that theaflavins affect not only the circulatory system but also skeletal muscle metabolism.

In the present study, we examined the effect of repeated oral administration of theaflavins on disuse muscle atrophy induced by hindlimb suspension in mice, and also assessed the protein levels involved in proteosynthesis and proteolysis in their hindlimb skeletal muscles.

\section{Materials and Methods}

Materials. Crude theaflavins fraction was obtained from reaction if green tea catechin extract and polyphenol oxidase. theaflavins were further purified by HPLC. Briefly, crude theaflavins fraction was eluted with $15 \%$ methanol and $15 \%$ acetonitrile by using YMC-DispoPackAT ODS column (YMC Co. Ltd., Kyoto, Japan) after removing catechins and caffeine with a gradient mobile phase of 3 to $6 \%$ methanol containing $0.1 \%$ phosphoric acid. The concentrations of theaflavins were determined by using HPLC as shown below; HPLC system, Series LC-20 (Shimazu, Kyoto, Japan); Column, Cadenza CL-C18 $(100 \times 4.6 \mathrm{~mm}, 3 \mu \mathrm{m}$, Imtakt Corporation, Kyoto, Japan); mobile phase, $20 \%$ acetonitrile with $0.1 \%$ formic acid; flow rate, $1 \mathrm{ml} / \mathrm{min}$; column temp, $40^{\circ} \mathrm{C}$; Detection, $280 \mathrm{~nm}$. The fraction contained $98.4 \%$ theaflavins and the composition was as follows; theaflavin, $38.1 \%$; theaflavin-3-O-gallate, $37.0 \%$; theaflavin 3 '- $O$-gallate, $11.0 \%$; and theaflavin-3,3'-di- $O$-gallate, $12.3 \%$. Unless otherwise noted, the chemicals using this study were purchased from FUJIFILM Wako Pure Chemical Corporation (Osaka, Japan).

Animals and diets. The study was approved by the Animal Care and Use Committee of the Shibaura Institute of Technology (Permit Number: 27-2956). All mice received humane care under

*To whom correspondence should be addressed. E-mail: nao-osa@shibaura-it.ac.jp 
the National Institutes of Health guide for the care and use of Laboratory animals in this institution. All surgery was performed under anesthesia, and all efforts were made to minimize suffering. Male C57BL/6J mice aged 17 to 19 weeks were obtained from Charles River Laboratories Japan, Inc. (Tokyo, Japan). The mice were kept in a room with controlled lighting $(12 / 12 \mathrm{~h}$ light/dark cycles) at a regulated temperature of $23-25^{\circ} \mathrm{C}$. A certified rodent diet (MF) was obtained from Oriental Yeast Company Limited (Tokyo, Japan).

Animal experiment. Thirty-two mice were fed a basal diet for 7 days and divided randomly into four groups as follows: ground-vehicle treatment, ground-theaflavins treatment, suspensionvehicle treatment, and suspension-theaflavins treatment. Mice in the vehicle treatment groups were administered $20 \%$ glycerol orally in saline, whereas animals in theaflavins treatment groups were dosed with theaflavins (gavage administration, $250 \mathrm{mg} / \mathrm{kg}$ body weight/day) dissolved in vehicle for 2 weeks. Hindlimb unloading was performed as described previously. ${ }^{(14)}$ Briefly, the animals in hindlimb suspension groups were elevated to a spinal orientation of $40-45^{\circ}$ above horizontal using a clip for tail suspension (Yamashita Giken, Tokushima, Japan) equipped with a block which was movable in a vertical direction. The proximal twothirds of the tail of the animals was wrapped with orthopedic traction tape. Hindlimb elevation was adjusted so that the hindlimb paws were suspended, while the forelimbs were free to ambulate around the entire range of the cage. At the end of this treatment period, the animals were sacrificed under anesthesia with $50 \mathrm{mg} / \mathrm{kg}$ s.c. pentobarbital (Tokyo Chemical Industry, Tokyo, Japan). Soleus (SOL), extensor digitorum longus (EDL), gastrocnemius (GASTRO) and tibialis anterior (TA) were removed, weighed and stored at $-80^{\circ} \mathrm{C}$. For histological analysis, SOL and EDL were blocked with FSC 22 Blue (LEICA, Tokyo, Japan) and frozen with isopentane on dry ice and stored at $-80^{\circ} \mathrm{C}$ until sectioning.

Histological analyses. All samples blocked with FSC were cut into $8 \mu \mathrm{m}$-thick sections using the LEICA CM1950 (LEICA, Wetzlar, Germany), and sections were stained with hematoxylin-eosin (HE) staining. Microscopic observation was carried out with an Olympus CX41 light microscope (Olympus Co., Tokyo, Japan). The muscle cross-sectional area (CSA) was quantified using ImageJ software (http://rsb.info.nih.gov/ij/ index.html, 11 June 2019).

Western blotting. The SOL and EDL were homogenized in a microtube with lysis buffer (CelLytic MT cell lysis reagent; Sigma-Aldrich, St. Louis, MO) containing a protease inhibitor (Sigma-Aldrich) and ( \pm )-dithiothreitol (FUJIFILM Wako Pure Chemical Corporation) using a Qsonic Model XL-2000 Series Sonicator (Arc Scientific, Los Angeles, CA). Protein concentrations were determined by Coomassie Blue staining (Thermo
Fisher Scientific, Waltham, MA). Protein $(50 \mu \mathrm{g})$ was separated by SDS-PAGE using a $5-12 \%$ and $10-20 \%$ Bis-Tris gel and transferred onto a $0.45 \mu \mathrm{m}$ and $0.2 \mu \mathrm{m}$ pore size polyvinylidene difluoride membrane (Life Technologies, Carlsbad, CA). The membrane was blocked with membrane-blocking reagent (GE Healthcare, Buckinghamshire, UK) for $1 \mathrm{~h}$. After being blocked, the membrane was incubated with a rabbit polyclonal primary antibody against phospho-Akt (1:1,000; ab1283, Abcam, Cambridge, UK) or Akt $(1: 2,000 ;$ ab28422, Abcam), phosphoeukaryotic translation initiation factor 4E-binding protein (4EBP-1, 1:1,000; \#2855, Cell Signalling Technology, Inc., Beverly, MA) or 4EBP-1 (1:1,000; \#9452, Cell Signalling Technology, Inc.), forkhead box (Fox) O3a $(1: 1,000 ; \# 2497$, Cell Signalling Technology, Inc.) or phospho-FoxO3a $(1: 1,000 ; \# 13129$, Cell Signalling Technology, Inc.) or Ubiquitin $(1: 1,000$; \#3933, Cell Signalling Technology, Inc.) under $4^{\circ} \mathrm{C}$ overnight. After the primary antibody reaction, the membrane was incubated with the horseradish peroxidase-conjugated secondary antibody $(1: 10,000)$ for $1 \mathrm{~h}$. Immunoreactivity was detected by chemiluminescence using the ECL Select Western Blotting Reagent (GE Healthcare, Madison, WI). Chemiluminescence band images were detected and analyzed by the C-DiGit Blot Scanner (LI-COR technology, Lincoln, NE).

Statistical analyses. All data were reported as mean $\pm \mathrm{SD}$. Statistical analyses were performed by two-way ANOVA followed by post hoc comparisons between experimental groups using Dunnett's test, or non-parametric Wilcoxon and MannWhitney $U$ tests. A probability of $p<0.05$ was considered to be statistically significant.

\section{Results}

There were no significant differences in body weight or tissue weight of liver, heart, kidney, adrenal gland or spleen of the experimental animals as shown in Table 1. The weight of SOL, GASTRO, TA and EDL in both hindlimb suspension groups (vehicle or theaflavins treatment group) were significantly decreased compared with ground-vehicle group.

We carried out the histological observation on SOL and EDL (Fig. 1 and 2). The distribution of myofiber size in SOL was determined as shown in Fig. 1B. The peak of myotube size was significantly moved to the smaller side in the suspension-vehicle group compared with the ground-vehicle group. This shift was significantly reduced by treatment with theaflavins (Fig. 1B and Table 2). The peak of myotube size of EDL also significantly moved to the smaller side in the suspension-vehicle group compared with the ground-vehicle group. This shift tended to be reduced by treatment with theaflavins (Fig. 2B and Table 2).

No significant changes were observed between experimental

Table 1. Body weight, tissue weight, and hindlimb skeletal muscle weight after repeated oral adminisrtrationadministration of vehicle or theaflavins with or without hindlimb suspention

\begin{tabular}{|c|c|c|c|c|}
\hline & \multicolumn{2}{|c|}{ Ground } & \multicolumn{2}{|c|}{ Suspension } \\
\hline & Vehicle & Theaflavins & Vehicle & Theaflavins \\
\hline Body weight (g) & $28.05 \pm 1.05$ & $27.72 \pm 0.66$ & $25.97 \pm 1.43$ & $25.58 \pm 1.69$ \\
\hline Heart (mg) & $123.4 \pm 15.2$ & $122.0 \pm 7.8$ & $120.1 \pm 6.2$ & $124.2 \pm 14.5$ \\
\hline Liver (mg) & $1,169.3 \pm 101.0$ & $1,222.5 \pm 72.7$ & $1,222.5 \pm 72.7$ & $1,097.6 \pm 82.5$ \\
\hline Spleen (mg) & $70.9 \pm 11.1$ & $63.0 \pm 3.5$ & $63.5 \pm 16.4$ & $62.1 \pm 14.0$ \\
\hline Kidney (mg) & $319.5 \pm 29.2$ & $299.6 \pm 9.3$ & $311.1 \pm 26.0$ & $328.6 \pm 25.7$ \\
\hline Adrenal grande $(\mathrm{mg})$ & $5.6 \pm 0.9$ & $4.8 \pm 0.8$ & $4.8 \pm 0.7$ & $4.6 \pm 0.5$ \\
\hline Soleus (mg) & $9.1 \pm 0.7$ & $8.9 \pm 0.6$ & $6.0 \pm 0.5 * *$ & $6.6 \pm 0.8$ ** \\
\hline Gastrocnemius (mg) & $163.4 \pm 10.9$ & $161.9 \pm 4.5$ & $136.0 \pm 6.6^{* *}$ & $137.4 \pm 8.1$ ** \\
\hline Tibialis anterior $(\mathrm{mg})$ & $51.1 \pm 3.2$ & $48.6 \pm 5.3$ & $43.0 \pm 2.8^{* *}$ & $42.6 \pm 3.5^{* *}$ \\
\hline Extensor digitorum longus (mg) & $13.6 \pm 1.0$ & $12.4 \pm 0.8$ & $11.3 \pm 0.8^{* *}$ & $11.5 \pm 0.9 * *$ \\
\hline
\end{tabular}

Each value represents the mean and SD $\left(n=6\right.$, each). Significantly different from the ground-vehicle group: ${ }^{* *} p<0.01$. 


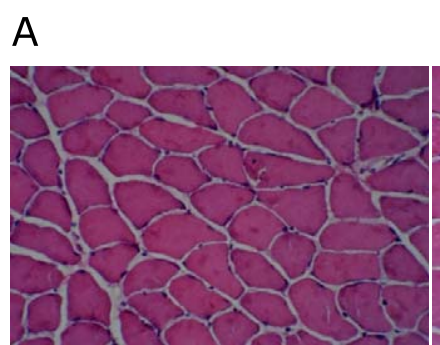

Vehicle

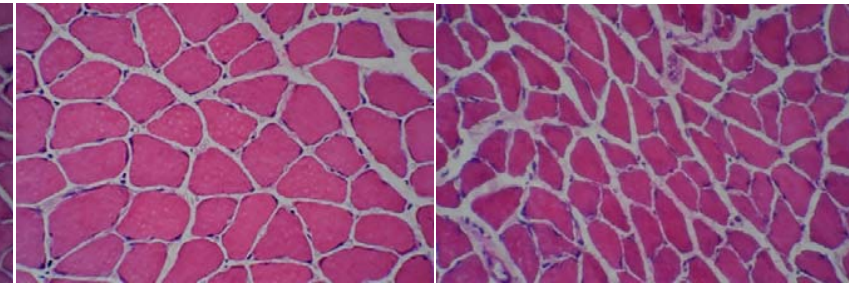

Theaflavins

Vehicle

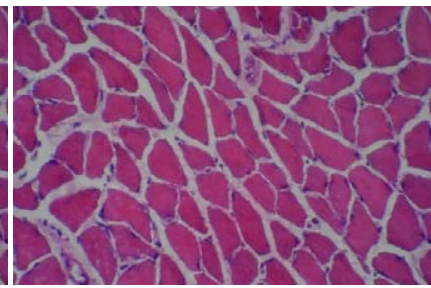

Theaflavins

Ground

Suspension

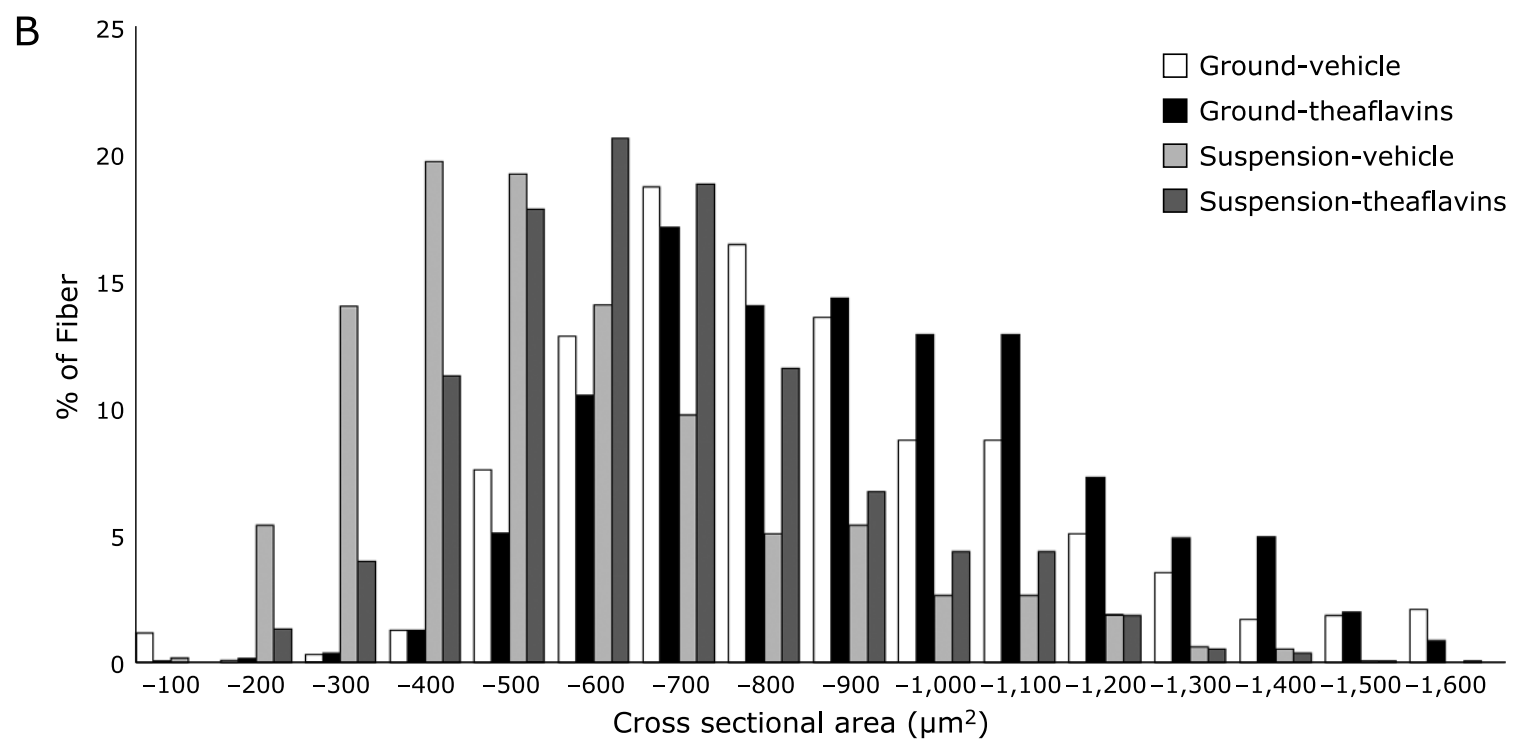

Fig. 1. The evaluation of skeletal muscle atrophy induced by hindlimb suspension with or without theaflavins; representative myofiber cross section of SOL stained with HE (A) and distribution of cross-sectional area of SOL (B). Statistical analyses were performed by Wilcoxon and MannWhitney $U$ tests (Table 2).

groups in phosphorylation of Akt level in SOL (Fig. 3). 4EBP-1 phosphorylation was not altered in ground groups, and there was a significant difference between suspension-vehicle and suspensiontheaflavins (Fig. 3). Dephosphorylation of FoxO3a was significantly increased in suspension-vehicle compared with groundvehicle group. These changes were reduced by treatment with theaflavins (Fig. 3). There were no significant changes in the level of ubiquitin between experimental groups in SOL (Fig. 3). There was no significant changes in phosphorylation of Akt in EDL (Fig. 4). 4EBP-1 phosphorylation was not altered in the ground groups, and it slightly increased in the suspension-theaflavins group (Fig. 4). Dephosphorylation of FoxO3a tended to increase in EDL in the suspension-vehicle group compared with the ground-vehicle group. This change was significantly reduced by the treatment with theaflavins (Fig. 4). There were no significant changes in the level of ubiquitin between experimental groups in EDL (Fig. 4).

\section{Discussion}

An interim hindlimb suspension period was reported to cause significant muscle atrophy in mammals. ${ }^{(5)}$ We found significant muscle atrophy in all skeletal muscles in hindlimb after 14 days of tail suspension (Table 1). These changes were more prominent in SOL with rich in slow muscle fibers than GASTRO, EA, and EDL. It was reported that rodent muscles that express predomi- nantly slow motor units reported to be more sensitive to the unloading stimuli compared to muscles expressing primarily fast motor units. ${ }^{(15)}$ In addition, these atrophy were reduced by the treatment with theaflavins (Fig. 1A and $2 \mathrm{~A}$ ). The peak of myotube size was significantly moved to the smaller side in the suspensionvehicle group compared with the ground-vehicle group, and these shifts were significantly reduced by the treatment with theaflavins in both SOL and EDL (Fig. 1B, 2B, and Table 2).

It was known that skeletal muscles adapt to change in their workload by regulating fibre size by both Akt/mTOR, anabolic signal, and FoxO pathway, catabolic signal. ${ }^{(16)}$ The Akt/mTOR pathway acts as a key regulator in the translation initiation step of protein synthesis in skeletal muscle. Under normal physiological conditions, it is activated by insulin like factor (IGF-1); therefore stimulated PI3K activates Akt. Phosphorylated Akt further activates $\mathrm{mTORC} 1$, resulting in activated mTORC1 phosphorylating both 4EBP-1 and ribosomal protein S6 kinase $\beta-1$, which ultimately leads to proteosynthesis. ${ }^{(17)}$ In contrast, IGF-1/ $\mathrm{PI} 3 \mathrm{~K} / \mathrm{Akt}$ signaling reduces unloading and joint immobilizationinduced muscle atrophy. ${ }^{(16)}$ In the present study, the level of phosphorylation of 4EBP-1 was higher in suspension-theaflavins compared with suspension-vehicle both in SOL and EDL (Fig. 3 and 4). According to these results, it was suggested that repeated oral treatment with theaflavins induces phosphorylation of 4EBP-1 as a result of the anabolic Akt/mTOR pathway. It is well investigated that FoxO3a controls transcriptional regulation 

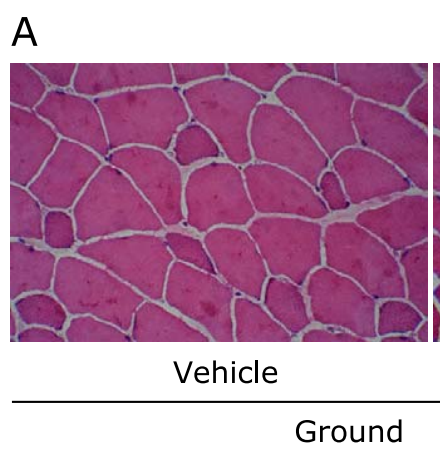

B

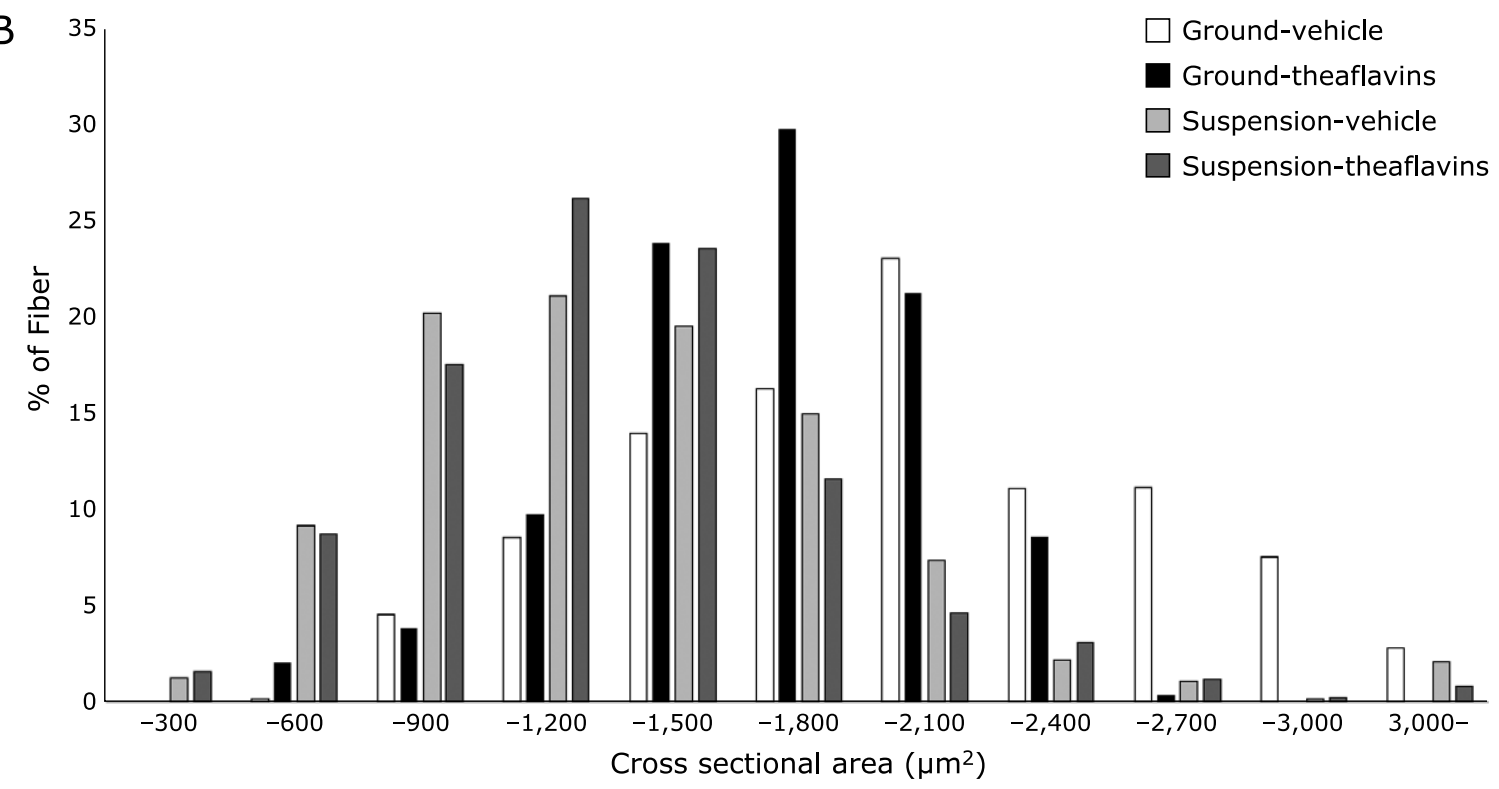

Fig. 2. The evaluation of skeletal muscle atrophy induced by hindlimb suspension with or without theaflavins; representative myofiber cross section of EDL stained with HE (A) and distribution of cross-sectional area of EDL (B). Each value represents mean and SD ( $n=6$, each). Statistical analyses were performed by Wilcoxon and Mann-Whitney $U$ tests (Table 2).

Table 2. Statistical results between experimental groups of the cross-sectional area in SOL (A) or EDL (B)

(A) SOL

\begin{tabular}{|c|c|c|c|c|c|c|c|c|c|c|c|c|c|c|c|c|c|}
\hline & \multirow{2}{*}{ Group } & \multicolumn{16}{|c|}{ Cross-Sectional Area $\left(\mu \mathrm{m}^{2}\right)$} \\
\hline & & -100 & -200 & -300 & -400 & -500 & -600 & -700 & -800 & -900 & $-1,000$ & $-1,100$ & $-1,200$ & $-1,300$ & $-1,400$ & $-1,500$ & $-1,600$ \\
\hline \multirow[t]{3}{*}{$\begin{array}{l}\text { Ground- } \\
\text { vehicle }\end{array}$} & $\begin{array}{l}\text { vs ground- } \\
\text { theaflavins }\end{array}$ & 0.182 & 1.000 & 0.849 & 1.000 & 0.699 & 0.699 & 0.485 & 0.589 & 0.937 & 0.394 & 0.310 & 0.485 & 0.191 & 0.645 & 0.303 & 0.849 \\
\hline & $\begin{array}{l}\text { vs suspension- } \\
\text { vehicle }\end{array}$ & 1.000 & $0.002 * *$ & $0.002 * *$ & $0.002 * *$ & $0.015^{*}$ & 0.937 & $0.041 *$ & 0.240 & $0.015^{*}$ & $0.026^{*}$ & $0.041 *$ & 0.370 & 0.546 & 0.103 & 0.455 & 0.455 \\
\hline & $\begin{array}{l}\text { vs suspension- } \\
\text { theaflavins }\end{array}$ & 0.455 & 0.303 & $0.019 *$ & $0.002 * *$ & $0.041 *$ & $0.041 *$ & 0.699 & 0.589 & $0.009 * *$ & 0.180 & $0.026 *$ & $0.039 *$ & 0.513 & 0.168 & 1.000 & 0.455 \\
\hline \multirow[t]{2}{*}{$\begin{array}{l}\text { Suspension- } \\
\text { vehicle }\end{array}$} & $\begin{array}{l}\text { vs ground- } \\
\text { theaflavins }\end{array}$ & 0.182 & 0.002 & $0.002 * *$ & $0.002 * *$ & $=0.002 * *$ & 0.310 & $0.041 *$ & $0.009 * *$ & $0.015^{*}$ & $0.004 * *$ & $0.009 * *$ & $0.091^{\#}$ & $0.058^{\#}$ & 0.182 & 0.697 & 0.455 \\
\hline & $\begin{array}{l}\text { vs suspension- } \\
\text { theaflavins }\end{array}$ & 0.692 & $0.041 *$ & 0.699 & $0.026^{*}$ & 0.818 & $0.009 * *$ & $0.009 * *$ & $0.015^{*}$ & 0.485 & 0.394 & 0.859 & 0.987 & 1.000 & 1.000 & 0.455 & 1.000 \\
\hline
\end{tabular}

(B) EDL

\begin{tabular}{|c|c|c|c|c|c|c|c|c|c|c|c|c|}
\hline & \multirow{2}{*}{ Group } & \multicolumn{11}{|c|}{ Cross-Sectional Area $\left(\mu \mathrm{m}^{2}\right)$} \\
\hline & & -300 & -600 & -900 & $-1,200$ & $-1,500$ & $-1,800$ & $-2,100$ & $-2,400$ & $-2,700$ & $-3,000$ & 3,000 \\
\hline \multirow[t]{3}{*}{$\begin{array}{l}\text { Ground- } \\
\text { vehicle }\end{array}$} & $\begin{array}{l}\text { vs ground- } \\
\text { theaflavins }\end{array}$ & 0.307 & 0.937 & 0.240 & 0.310 & 0.818 & 0.818 & 0.208 & $0.099^{\#}$ & 0.697 & 1.000 & 0.455 \\
\hline & $\begin{array}{l}\text { vs suspension- } \\
\text { vehicle }\end{array}$ & $0.015^{*}$ & $0.026^{*}$ & $0.002 * *$ & 0.485 & $0.09^{\#}$ & $0.004 * *$ & 0.208 & $0.060^{\#}$ & 0.455 & 1.000 & 1.000 \\
\hline & $\begin{array}{l}\text { vs suspension- } \\
\text { theaflavins }\end{array}$ & 0.582 & 0.240 & $0.002 * *$ & $0.015^{*}$ & 0.180 & $0.015^{*}$ & 0.208 & $0.060^{\#}$ & 0.455 & 1.000 & 0.455 \\
\hline \multirow[t]{2}{*}{$\begin{array}{l}\text { Suspension- } \\
\text { vehicle }\end{array}$} & $\begin{array}{l}\text { vs ground- } \\
\text { theaflavins }\end{array}$ & $0.026^{*}$ & $0.026^{*}$ & $0.002 * *$ & 0.937 & 0.026 * & $0.067^{\#}$ & 1.000 & 0.455 & 0.455 & 1.000 & 1.000 \\
\hline & $\begin{array}{l}\text { vs suspension- } \\
\text { theaflavins }\end{array}$ & $0.026 *$ & 0.180 & 0.818 & $0.064^{\#}$ & 0.309 & $0.093^{\#}$ & 0.922 & 1.000 & 1.000 & 1.000 & 0.455 \\
\hline
\end{tabular}

Each number represents $p$ value performed by non-parametric Wilcoxon and Mann-Whitney $U$ tests. ${ }^{\#} p<0.1,{ }^{*} p<0.05,{ }^{*} p<0.01$. 


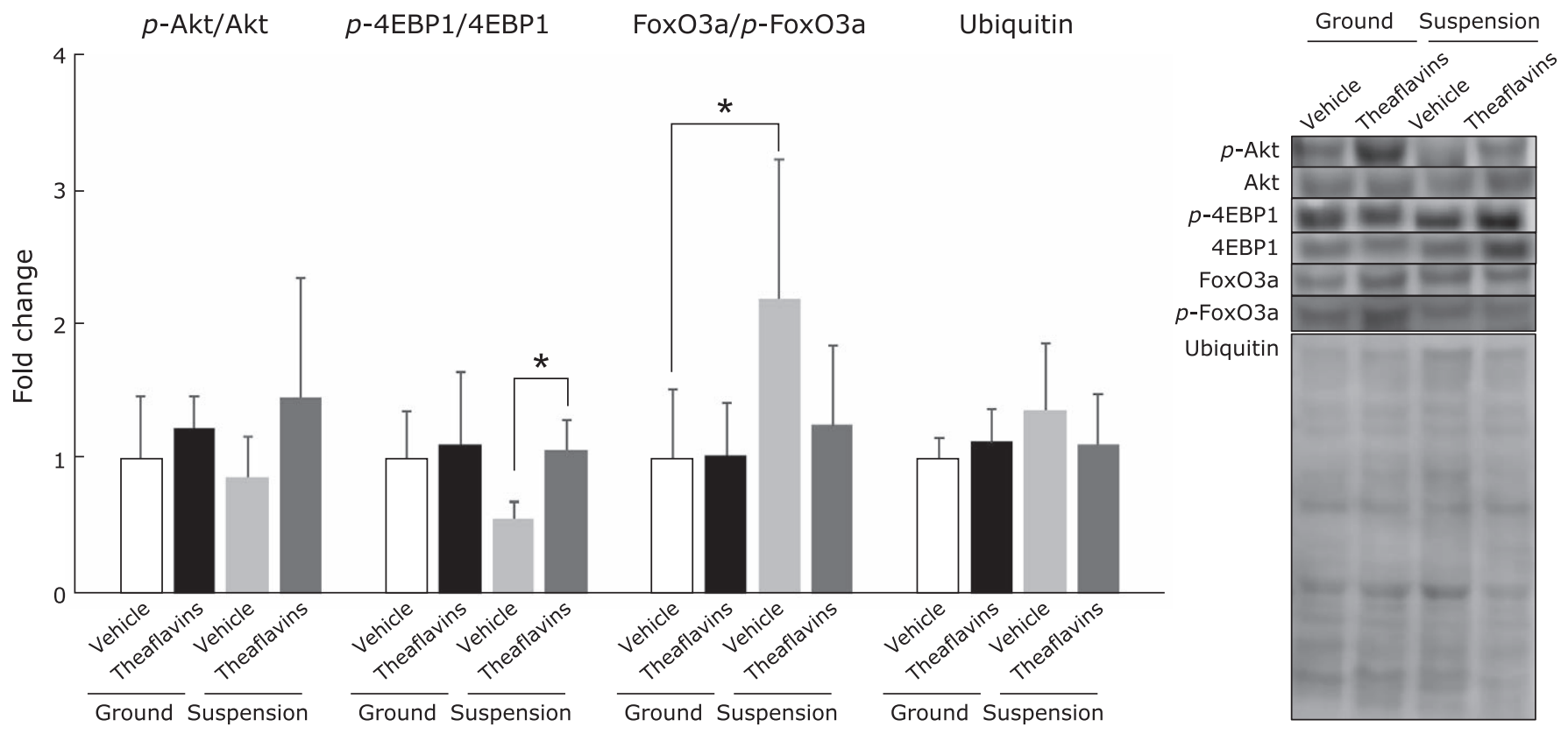

Fig. 3. Ratio of phosphorylated Akt to Akt, phosphorylated 4EBP-1 to 4EBP-1, FoxO 3a to phosphorylated FoxO and ubiquitin of SOL. Each value represents mean and SD ( $n=6$, each). Statistical analyses were performed by two-way ANOVA followed by Dunnett's test. Significant differences between experimental groups; ${ }^{*} p<0.05$.

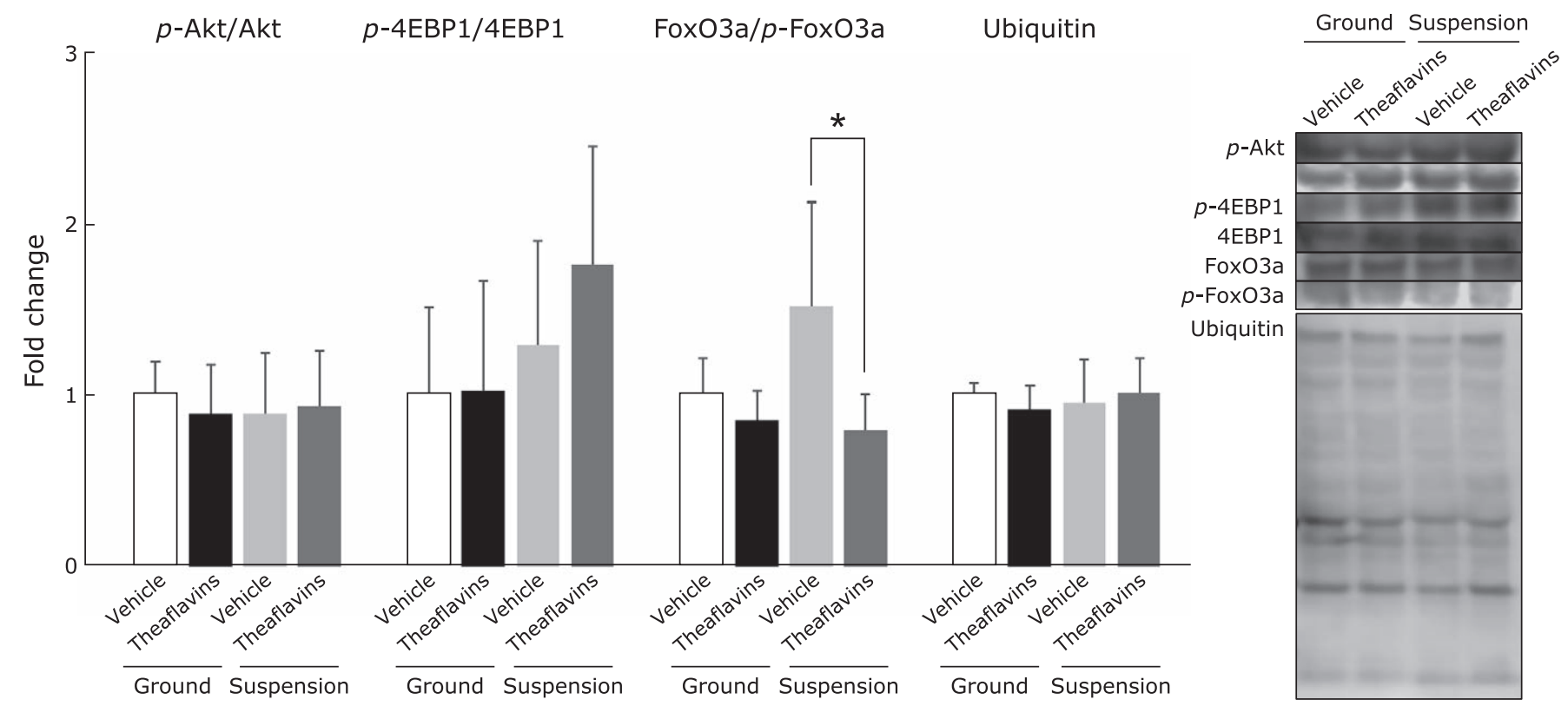

Fig. 4. Ratio of phosphorylated Akt to Akt, phosphorylated 4EBP-1 to 4EBP-1, FoxO 3a to phosphorylated FoxO and ubiquitin of EDL. Each value represents mean and SD $(n=6$, each). Statistical analyses were performed by two-way ANOVA followed by Dunnett's test. Significant difference between experimental groups; ${ }^{*} p<0.05$.

of muscle-specific ubiquitin ligase when the disuse atrophy progresses. ${ }^{(18)} \mathrm{We}$ confirmed that the FoxO3a/p-FoxO3a ratio was significantly increased in SOL and tended to increase in EDL by the hindlimb suspension. These changes were reduced by the administration of theaflavins (Fig. 3 and 4). These results suggested that repeated supplementation of theaflavins inhibited the activation of the catabolic FoxO pathway.

In previous studies, it was reported that oral administration of 100 to $200 \mathrm{mg} / \mathrm{kg}$ theaflavins prevented hyperglycemia in diabetes model rats. ${ }^{(19,20)}$ It is well known that increased skeletal muscle metabolism promotes glucose uptake into skeletal muscle and improves hyperglycemia. Our present result supported these previous results, showing about almost similar amount of theaflavins promote skeletal muscle metabolism.

In addition, a regular cup of black tea contains $\sim 16-24 \mathrm{mg}$ of theaflavins per serving of $200-230 \mathrm{ml} .^{(21)}$ It was also reported that drinking four or more cups of black tea on a daily basis inhibited the risk associated with stroke by an extensively study 


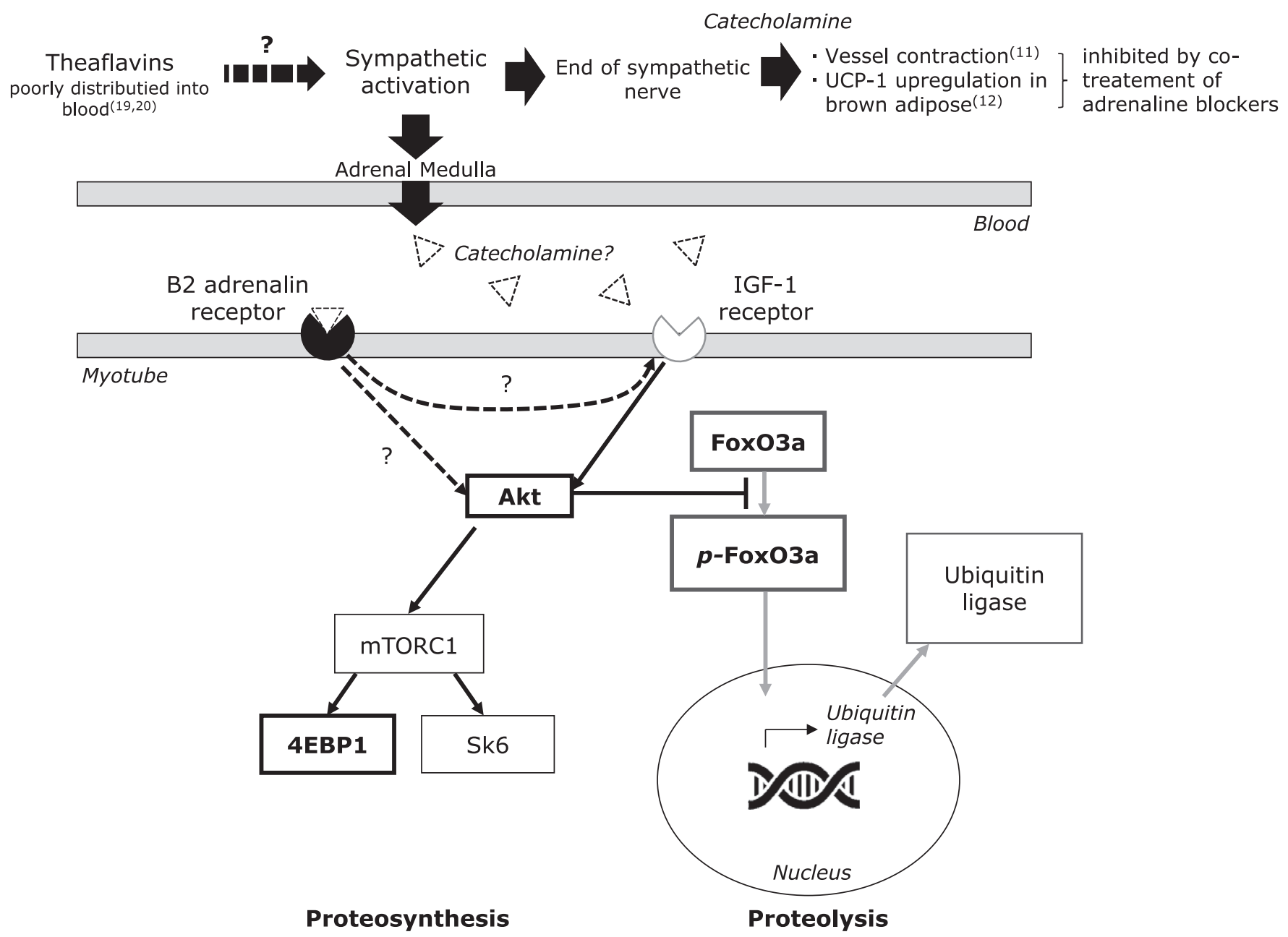

Fig. 5. Possible mechanism of action of theaflavins on the disuse muscle atrophy.

on 74,961 stroke patients during the course of 10.2 years. $^{(22)}$ However, the effective dose of theaflavin for reducing the risk of stroke has not been elucidated. As one of the causes, several methods have been developed for measuring theaflavins in blood or tissues, ${ }^{(23,24)}$ but there was limited research on the theaflavins concentration in blood after theaflavins supplementation. These studies suggested that theaflavins had relatively low bioavailability compared with other polyphenols. Despite this poor bioavailability, theaflavins has been reported to cause significant primary prevention of cardiovascular diseases by decreasing plasma LDL cholesterol levels and blood pressure. ${ }^{(9,10)}$ In addition, a single oral dose of theaflavins as black tea was reported to improve vascular endothelial function in healthy volunteers. ${ }^{(25,26)}$ In our previous report, we confirmed a similar improvement of vascular endothelial function, measuring skeletal muscle blood flow and phosphorylation of eNOS in mammalian aorta after a single dose of theaflavin mixture or four major theaflavins each. ${ }^{(11)}$ These changes were significantly reduced by treatment with an adrenalin blocker such as carvedilol. According to this result, it was suggested that an oral dose of theaflavins induced sympathetic nerve activation.

It is well known that the sympathetic nervous system plays a key role in physiological adaptation not only in the circulatory system but also in the metabolic system. ${ }^{(27,28)}$ Catecholamines, are secreted from the end of the sympathetic nerve or adrenal medulla, bind to adrenergic receptors, and maintains homeostasis by expressing various actions. In circulation, catecholamines tran- siently increases heart rate and blood presser in sympathetic hyperactivity and these changes are inhibited by the pretreatment of adrenalin blocker. Nonshivering thermogenesis is also exhibited in brown adipose tissue through activation of uncoupling protein 1by the sympathetic hyperactivity such as cold exposure, and this change is also inhibited by the treatment of adrenalin blocker. In our previous research, we confirmed that a single oral dose of theaflavins showed not only the transient hemodynamic changes but also nonshivering thermogenesis. ${ }^{(11,12)}$ During exercise, the increase of plasma catecholamines secreted from the adrenal medulla by sympathetic hyperactivity bind to skeletal muscle $\beta 2$ receptor and enhancing skeletal muscle protein synthesis through Akt/mTOR pathway. ${ }^{(29)}$ In addition, $\beta 2$ adrenaline agonists, such as formoterol or clenbuterol were reported to inhibited skeletal muscle atrophy and induce skeletal muscle hypertrophy. ${ }^{(30-33)}$ Taken together these findings, the effect of theaflavins on skeletal muscle atrophy showing in the present study was suggested as adrenergic activity (Fig. 5). However, further research is needed to elucidate how theaflavins induce sympathetic hyperactivity.

In conclusion, we found that repeated doses of theaflavins reduced disuse muscle atrophy induced by hindlimb suspension. It was suggested that repeated oral doses of theaflavins modulated protein metabolism of skeletal muscle. Further experiments are needed to elucidate the mechanisms, including the adrenomimetic activity of theaflavins. 


\section{Author Contributions}

KS and NH conducted experiments. YF, TF, and RA performed data analysis and interpretation of data. AY and TU provide research source. KS, AY, and NO wrote the initial draft of the manuscript. TU and NO designed the study. NO critically reviewed the manuscript. All authors approved the final version of the manuscript and agree to be accountable for all aspects of the work in ensuring that questions related to the accuracy or integrity of any part of the work are appropriately investigated and resolved.

\section{Aknowledgments}

This work was supported by Yaizu Suisankagaku Ind. Co. Ltd.

\section{Abbreviations}

AMPK AMP-activated protein kinase

4EBP 4E-binding protein

EDL extensor digitorum longus

GASTRO gastrocnemius

HE hematoxylin-eosin

PGC-1 $\alpha$ proliferator-activated receptor gamma coactivator- $1 \alpha$

SOL soleus

TA tibialis anterior

\section{Conflict of Interest}

This study was funded by Yaizu Suisankagaku Ind. Co. Ltd. AY and TU are employees of Yaizu Suisankagaku Ind. Co. Ltd. and provide research source. $\mathrm{KS}, \mathrm{NH}, \mathrm{YF}, \mathrm{TF}, \mathrm{RA}$, and NO declare no competing interests.

\section{References}

1 Rudrappa SS, Wilkinson DJ, Greenhaff PL, Smith K, Idris I, Atherton PJ. Human skeletal muscle disuse atrophy: effects on muscle protein synthesis, breakdown, and insulin resistance - a qualitative review. Front Physiol 2016; 7: 361 .

2 Magne H, Savary-Auzeloux I, Rémond D, Dardevet D. Nutritional strategies to counteract muscle atrophy caused by disuse and to improve recovery. Nutr Res Rev 2013; 26: 149-165.

3 Millward DJ, Garlick PJ, Stewart RJ, Nnanyelugo DO, Waterlow JC. Skeletal-muscle growth and protein turnover. Biochem J 1975; 150: 235-243.

4 Powers SK. Can antioxidants protect against disuse muscle atrophy? Sports Med 2014; 44 Suppl 2: S155-S165.

5 Brooks NE, Myburgh KH. Skeletal muscle wasting with disuse atrophy is multi-dimensional: the response and interaction of myonuclei, satellite cells and signaling pathways. Front Physiol 2014; 5: 99.

6 Tsika RW, Herrick RE, Baldwin KM. Effect of anabolic steroids on skeletal muscle mass during hindlimb suspension. J Appl Physiol (1985) 1987; 63: 2122-2127.

7 Haslam E. Thoughts on thearubigins. Phytochemistry 2003; 64: 61-73.

8 Sang S, Lambert JD, Ho CT, Yang CS. The chemistry and biotransformation of tea constituents. Pharmacol Res 2011; 64: 87-99.

9 Hartley L, Flowers N, Holmes J, et al. Green and black tea for the primary prevention of cardiovascular disease. Cochrane Database Syst Rev 2013; 2013: CD009934.

10 Santesso N, Manheimer E. A summary of a cochrane review: green and black tea for the primary prevention of cardiovascular disease. Glob Adv Health Med 2014; 3: 66-67.

11 Saito A, Nakazato R, Suhara Y, et al. The impact of theaflavins on systemicand microcirculation alterations: the murine and randomized feasibility trials. J Nutr Biochem 2016; 32: 107-114.

12 Kudo N, Arai Y, Suhara Y, Ishii T, Nakayama T, Osakabe N. A single oral administration of theaflavins increases energy expenditure and the expression of metabolic genes. PLoS One 2015; 10: e0137809.

13 Aizawa T, Yamamoto A, Ueno T. Effect of oral theaflavin administration on body weight, fat, and muscle in healthy subjects: a randomized pilot study. Biosci Biotechnol Biochem 2017; 81: 311-315.

14 Morey-Holton ER, Globus RK. Hindlimb unloading rodent model: technical aspects. J Appl Physiol (1985) 2002; 92: 1367-1377.

15 Schiaffino S, Reggiani C. Molecular diversity of myofibrillar proteins: gene regulation and functional significance. Physiol Rev 1996; 76: 371-423.

16 Gao Y, Arfat Y, Wang H, Goswami N. Muscle atrophy induced by mechanical unloading: mechanisms and potential countermeasures. Front Physiol 2018; 9: 235

17 Sharples AP, Hughes DC, Deane CS, Saini A, Selman C, Stewart CE. Longevity and skeletal muscle mass: the role of IGF signalling, the sirtuins, dietary restriction and protein intake. Aging Cell 2015; 14: 511-523.

18 Sandri M, Lin J, Handschin C, et al. PGC-1alpha protects skeletal muscle from atrophy by suppressing FoxO3 action and atrophy-specific gene transcription. Proc Natl Acad Sci U S A 2006; 103: 16260-16265.

19 Gothandam K, Ganesan VS, Ayyasamy T, Ramalingam S. Antioxidant

potential of theaflavin ameliorates the activities of key enzymes of glucose metabolism in high fat diet and streptozotocin-induced diabetic rats. Redox Rep 2019; 24: 41-50.

20 Kobayashi M, Ichitani M, Suzuki Y, et al. Black-tea polyphenols suppress postprandial hypertriacylglycerolemia by suppressing lymphatic transport of dietary fat in rats. J Agric Food Chem 2009; 57: 7131-7136.

21 Vermeer MA, Mulder TP, Molhuizen HO. Theaflavins from black tea, especially theaflavin-3-gallate, reduce the incorporation of cholesterol into mixed micelles. J Agric Food Chem 2008; 56: 12031-12036.

22 Arab L, Liu W, Elashoff D. Green and black tea consumption and risk of stroke: a meta-analysis. Stroke 2009; 40: 1786-1792.

23 Neilson AP, Green RJ, Wood KV, Ferruzzi MG. High-throughput analysis of catechins and theaflavins by high performance liquid chromatography with diode array detection. $J$ Chromatogr A 2006; 1132: 132-140.

24 Nishimura M, Ishiyama K, Watanabe A, Kawano S, Miyase T, Sano M. Determination of theaflavins including methylated theaflavins in black tea leaves by solid-phase extraction and HPLC analysis. J Agric Food Chem 2007; 55: 7252-7257.

25 Jochmann N, Lorenz M, von Krosigk A, et al. The efficacy of black tea in ameliorating endothelial function is equivalent to that of green tea. Br J Nutr 2008; 99: 863-868.

26 Grassi D, Mulder TP, Draijer R, Desideri G, Molhuizen HO, Ferri C. Black tea consumption dose-dependently improves flow-mediated dilation in healthy males. J Hypertens 2009; 27: 774-781.

27 Fongemie J, Felix-Getzik E. A review of nebivolol pharmacology and clinical evidence. Drugs 2015; 75: 1349-1371.

28 Axelrod J, Weinshilboum R. Catecholamines. N Engl J Med 1972; 287: 237 242.

29 Cairns SP, Borrani F. $\beta$-Adrenergic modulation of skeletal muscle contraction: key role of excitation-contraction coupling. J Physiol 2015; 593: 4713-4727.

30 Ryall JG, Schertzer JD, Lynch GS. Attenuation of age-related muscle wasting and weakness in rats after formoterol treatment: therapeutic implications for sarcopenia. J Gerontol A Biol Sci Med Sci 2007; 62: 813-823.

31 Ryall JG, Sillence MN, Lynch GS. Systemic administration of beta2adrenoceptor agonists, formoterol and salmeterol, elicit skeletal muscle hypertrophy in rats at micromolar doses. Br J Pharmacol 2006; 147: 587-595.

32 Sirvent P, Douillard A, Galbes O, et al. Effects of chronic administration of clenbuterol on contractile properties and calcium homeostasis in rat extensor digitorum longus muscle. PLoS One 2014; 9: e100281.

33 Yimlamai T, Dodd SL, Borst SE, Park S. Clenbuterol induces musclespecific attenuation of atrophy through effects on the ubiquitin-proteasome pathway. J Appl Physiol (1985) 2005; 99: 71-80.

This is an open access article distributed under the terms of the Creative Commons Attribution-NonCommercial-NoDerivatives License (http://creativecommons.org/licenses/by-nc-nd/4.0/). 